\title{
Sclerosing adenosis: Ultrasonographic and mammographic findings and correlation with histopathology
}

\author{
YA-LING CHEN $^{1,2^{*}}$, JIA-JIAN $\mathrm{CHEN}^{2,3^{*}}$, CAI CHANG $^{1,2}$, YI GAO $^{1,2}$, \\ JIONG WU ${ }^{2,3}$, WEN-TAO YANG ${ }^{2,4}$ and YA-JIA GU ${ }^{2,5}$ \\ ${ }^{1}$ Department of Ultrasound, Fudan University Shanghai Cancer Center; ${ }^{2}$ Department of Oncology, \\ Shanghai Medical College, Fudan University; Departments of ${ }^{3}$ Breast Surgery, ${ }^{4}$ Pathology and \\ ${ }^{5}$ Diagnostic Radiology, Fudan University Shanghai Cancer Center, Shanghai 200032, P.R. China
}

Received October 4, 2016; Accepted November 4, 2016

DOI: $10.3892 / \operatorname{mco} .2016 .1108$

\begin{abstract}
The present study was conducted to evaluate the radiological findings, particularly the ultrasonographic (US) characteristics of sclerosing adenosis (SA), and their correlation with histopathological results. A retrospective review identified 191 patients with a total of 200 lesions histopathologically confirmed as SA following breast surgery between July 2009 and December 2012. Of the 191 patients, 145 (151 lesions) with SA as the major component were included for US and mammographic (MG) analysis. All 145 patients analyzed were female, with a mean age \pm standard deviation of $46.8 \pm 7.8$ years (range, 25-71 years). All 145 patients underwent US examination and the imaging findings included heterogeneously echogenic areas in $9.3 \%$ $(14 / 151)$, masses in $51.7 \%(78 / 151)$, masses with calcifications in $13.9 \%(21 / 151)$, focal acoustic shadowing in $4.0 \%$ $(6 / 151)$ and were negative in $21.2 \%$ (32/151) patients. Among the 119 lesions with visible abnormalities, 87.4\% (104/119) were hypoechoic, $58.0 \%(69 / 119)$ were irregular in shape, $52.1 \%(62 / 119)$ had an ill-defined margin, calcifications were found in $17.6 \%(21 / 119)$ and $7.6 \%$ (9/119) were hypervascular, while none of the characteristics mentioned above were significantly correlated with histopathology. A total of 136 patients underwent MG at the Fudan University Shanghai Cancer Center, and the imaging findings included microcalcifications in $31.6 \%(43 / 136)$, masses in $23.5 \%$ (32/136), asymmetric focal density in $14.7 \%$ (20/136), focal architectural distortion in $22.8 \%$ (31/136), and were negative in $7.4 \%$ (10/136). The mass lesions were fewer on MG compared with US (23.5 vs. $65.6 \%$, respectively). The area
\end{abstract}

Correspondence to: Dr Cai Chang, Department of Ultrasound, Fudan University Shanghai Cancer Center, 270 Dong-An Road, Shanghai 200032, P.R. China

E-mail: changcai1962@163.com

*Contributed equally

Key words: breast, sclerosing adenosis, ultrasonography, mammography, histopathology under the curve of US distinguishing between benign and malignant lesions was significantly larger compared with that of MG (0.547 vs. 0.497 , respectively; $\mathrm{P}=0.036)$. In the 60 lesions that were overestimated by Breast Imaging Reporting and Data System US category, one or more characteristics of malignancy were found on US imaging. The most common finding of SA was masses with or without calcifications on US and microcalcifications on MG. The accuracy of US was limited, but higher compared with that of MG; however, SA mimicking the characteristics of malignancy may contribute to misdiagnosis with US.

\section{Introduction}

As a subtype of adenosis, sclerosing adenosis (SA) is a benign proliferative disease of the breast associated with disordered acinar, myoepithelial and connective tissue in the terminal ductal lobular unit. Despite its benign pathological behavior, SA may mimic in situ and invasive carcinoma grossly and microscopically $(1,2)$; thus further investigation is crucial to fully understand this disease and its imaging characteristics.

Several articles have described the clinical and imaging characteristics of SA over the past few decades (3-6). As reported in previous studies (5), a proportion of patients with SA complained of mastalgia (14.0\%), others were detected with a palpable mass $(30.2 \%)$, while several asymptomatic patients were identified through imaging examination, and the findings on mammography (MG) were mainly microcalcifications $(55.8 \%)$, mass (11.6\%), asymmetric focal density (6.9\%) and focal architectural distortion (6.9\%). However, only few studies have investigated the imaging characteristics of SA on ultrasonography (US).

In China, a higher number of individuals seek periodic breast examination due to the increasing incidence of breast diseases, and breast US, as a radiation-free modality, is the most popular screening method due to the advantages of high safety and low cost. In addition, Eastern women tend to have dense mammary glands, which may be more clearly displayed on US compared with MG. The objective of the present study was to focus on the radiological findings, particularly the US characteristics of SA, and to determine the correlation of these findings with histopathological results. 


\section{Patients and methods}

Patients. A total of 24,239 patients who underwent breast surgery between July, 2009 and December, 2012 at the Fudan University Cancer Center (Shanghai, China) were retrospectively investigated. SA was histopathologically diagnosed in a total of 191 female patients (200 lesions), among whom 145 patients (151 lesions) with SA as the major component were included for clinical and imaging analysis. In the remaining 46 patients (49 lesions), SA was an incidental pathological finding following surgery for malignant tumors (21 lesions in 19 patients) and benign tumors (28 lesions in 27 patients), and these cases were excluded from the study.

The present study was conducted following approval by the Institutional Review Board (IRB) of Fudan University Shanghai Cancer Center. Written informed consent was waived by the IRB due to the retrospective nature of the study. No study subjects or cohorts have been previously reported.

US. All 145 patients underwent US prior to surgery. All the examinations were performed by sonographers experienced in breast US with a linear array transducer (IU22, Philips, Bothell, WA, USA; and Mylab 90, Esaote, Genoa, Italy). A final assessment was made and each case was classified preoperatively based on the American College of Radiology Breast Imaging Reporting and Data System (BI-RADS) US lexicon criteria $(7,8)$. The morphology of the lesions, including size, shape, margin, internal echo pattern and posterior acoustic feature, was analyzed by two experienced investigators blinded to the study. Color Doppler US and power Doppler US were performed to evaluate the vessels within and surrounding the lesions.

$M G$. Among the 145 patients (151 lesions), 9 (15 lesions) underwent MG in other hospitals prior to admission to our center, and the mammograms of these patients were unavailable for analysis. The remaining 136 patients underwent MG in our center prior to surgery; craniocaudal and mediolateral oblique mammograms of both breasts were obtained using Senographe 2000D equipment (General Electrics, Detroit, MI, USA). The mammograms for each lesion were reviewed by three radiologists experienced in breast imaging and characterized according to mass size, characteristics, morphology and distribution of microcalcifications.

Correlation with histopathology. All the lesions were surgically excised. The non-palpable lesions were preoperatively localized by using a needle-wire system placed under MG (23 lesions in 23 patients) and US (52 lesions in 48 patients) guidance, and underwent specimen radiographs and US prints to confirm removal of the lesions.

To analyze the accuracy of US and MG diagnosing the cases as benign or malignant, the BI-RADS category was compared with the histopathological results. Categories 1-3 were classified as the benign group, while categories $4 \mathrm{~B}, 4 \mathrm{C}$ and 5 were classified as the malignant group. Despite a malignant probability of $<10 \%$, category $4 \mathrm{~A}$ was classified in the malignant group in our study, due to the indication of clinical intervention. Category 0 was also included in the malignant group due to the requirement for further examinations.
Statistical analysis. Statistical analyses were performed using SPSS software, version 19.0 (SPSS, Chicago, IL, USA). To evaluate diagnostic performance, receiver operating characteristic (ROC) curves were analyzed using MedCalc for Windows, version 13.1.2.0 (MedCalc Software, Mariakerke, Belgium). Sensitivity, specificity and area under the ROC curve (AUC) were calculated. Measurement data were analyzed with the t-test and count data were analyzed with the Chi-squared test. A P-value of $<0.05$ was considered to indicate a statistically significant difference.

\section{Results}

Patient characteristics. All the 145 patients analyzed were female, with a mean age \pm standard deviation of $46.8 \pm 7.8$ years (range, 25-71 years). None of the patients had a family history of breast cancer. In 6 patients (4.1\%), SA was found bilaterally; 18 patients (12.4\%) complained of mastalgia and 5 patients (3.4\%) complained of nipple discharge; 74 patients (51.0\%) reported awareness of a breast mass, while 48 patients (33.1\%) were asymptomatic.

All the lesions were surgically excised. Of the 151 lesions, 20 (13.4\%) were SA with malignant lesions as the minor component, among which 13 lesions were ductal carcinoma in situ (DCIS) with bilateral involvement in 1 patient, 4 lesions were DCIS with microinvasion (DCISM), and 3 lesions were invasive ductal carcinoma (IDC). A total of 58 lesions (38.4\%) were SA with benign lesions, such as ductal hyperplasia, fibroadenomas and intraductal papilloma, and the remaining 73 lesions $(48.3 \%)$ were pure SA.

US findings. All 145 patients (151 lesions) underwent US examination. Apart from the 32 lesions (21.2\%) without visible abnormalities, there were mainly four types of imaging findings: Heterogeneously echogenic areas $(n=14 ; 9.3 \%)$, mass lesions ( $\mathrm{n}=78 ; 51.7 \%)$, masses with calcifications $(\mathrm{n}=21 ; 13.9 \%)$ and focal acoustic shadowing $(n=6 ; 4.0 \%)$ (Fig. 1). The US images of these 119 visible lesions were reviewed and the characteristics were summarized as follows (Table I): $87.4 \%$ of the lesions (104/119) were hypoechoic, 58.0\% (69/119) were irregular in shape and 52.1\% (62/119) had an ill-defined margin. Among the mass lesions with or without calcifications, 90.9\% (90/99) were solid, 4.0\% (4/99) were cystic with thickened walls, and 5.1\% (5/99) were mixed. Calcifications were detected in 21 lesions (17.6\%), of which 9 were clustered punctate, 5 were diffusely punctate and 7 were clustered amorphous. According to the grading system reported by Adler et al (9), only 9 lesions (7.6\%) were hypervascular. However, none of the characteristics mentioned above was significantly correlated with pathological type $(\mathrm{P}>0.05)$.

Upon comparing BI-RADS US category with histopathology, the AUC of US distinguishing benign from malignant lesions was 0.547 . The sensitivity, specificity, positive predictive value and negative predictive value of indicating clinical intervention (BI-RADS categories 0 and 4A-5) were 44.4, $54.9,11.8$ and $88.0 \%$, respectively. Of the 23 lesions of reasonable and high suspicion of malignancy (4B-5), only 1 case was finally diagnosed as malignant, which was SA with IDC as the minor component. However, $85.3 \%$ of category $4 \mathrm{~A}$ cases were finally confirmed as benign (Table II). The false-positive 
Table I. Ultrasonographic findings of 119 solitary lesions in patients with sclerosing adenosis of the breast.

\begin{tabular}{lcr}
\hline Characteristics & $\begin{array}{c}\text { No. of } \\
\text { lesions (\%) }\end{array}$ & $\begin{array}{r}\text { Pearson } \chi^{2} \\
(\text { P-value })^{\mathrm{b}}\end{array}$ \\
\hline $\begin{array}{l}\text { Shape } \\
\text { Regular }\end{array}$ & $50(42.0)$ & $0.159(0.690)$ \\
Irregular & $69(58.0)$ & \\
Echo pattern & & $4.877(0.181)$ \\
Hypoechoic & $104(87.4)$ & \\
Isoechoic & $4(3.4)$ & \\
Hyperechoic & $2(1.7)$ & \\
Mixed echoic & $5(4.3)$ & \\
Echoless & $4(3.4)$ & \\
Margin & & $0.646(0.421)$ \\
Well-defined & $57(47.9)$ & \\
Ill-defined & $62(52.1)$ & \\
Calcifications & & $0.044(0.833)$ \\
Absent & $98(82.4)$ & \\
Present & $21(17.6)$ & \\
Focal acoustic shadowing & & $0.415(0.519)$ \\
Absent & $113(96.0)$ & \\
Present & $6(4.0)$ & \\
Vascularitya & & \\
None (grade 0) & $92(77.3)$ & \\
Hypovascular (grade I) & $18(15.1)$ & \\
Hypervascular (grade II-III) & $9(7.6 \%)$ & \\
\hline
\end{tabular}

aVascularity was graded according to the grading system reported by Adler et al (9). ${ }^{\text {b }}$ Correlation between ultrasonographic characteristics and pathology (benign or malignant lesion; $\mathrm{P}<0.05$ was considered to indicate statistically significant differences.

Table II. Performance of ultrasonography (US) and mammography $(\mathrm{MG})$ in sclerosing adenosis of the breast.

\begin{tabular}{lrc}
\hline & \multicolumn{2}{c}{ Histopathology of the lesions } \\
\cline { 2 - 3 } & Benign (n) & Malignant (n) \\
\hline BI-RADS US (151 lesions) & & \\
0 & 9 & 2 \\
1-3 & 73 & 10 \\
4A & 29 & 5 \\
4B-5 & 22 & 1 \\
Total & 133 & 18 \\
BI-RADS MG (136 lesions) & & \\
0 & 17 & 4 \\
1-3 & 41 & 4 \\
4A & 34 & 5 \\
4B-5 & 26 & 5 \\
Total & 118 & 18 \\
\hline
\end{tabular}

BI-RADS, Breast Imaging Reporting and Data System.

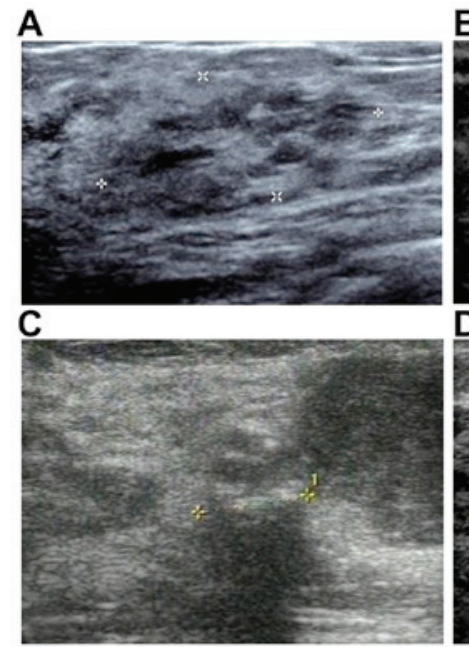

B

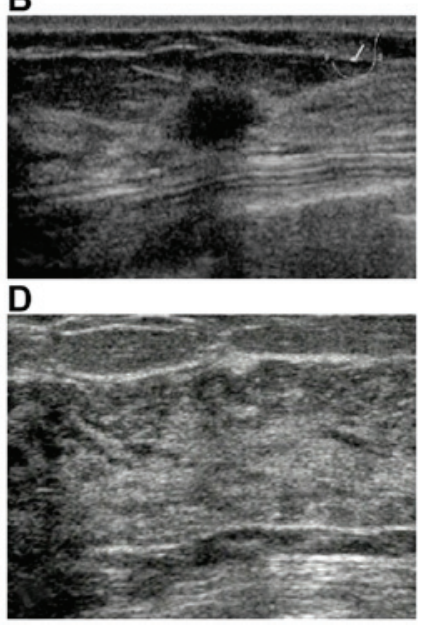

Figure 1. Ultrasonographic findings in sclerosing adenosis. (A) Heterogeneously echogenic areas; (B) mass lesions; (C) mass lesions with calcifications; and (D) focal acoustic shadowing.
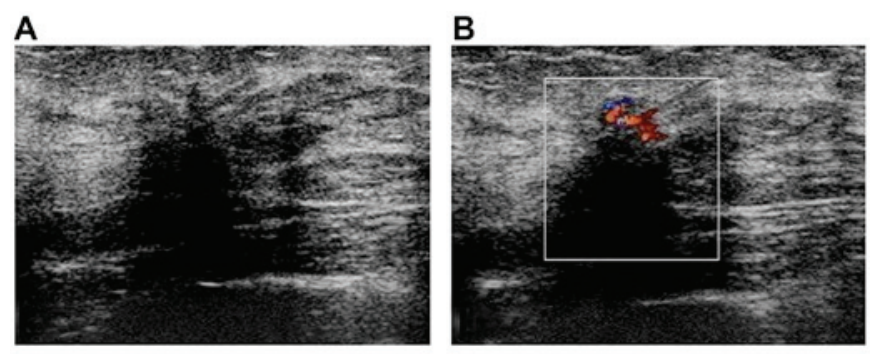

C
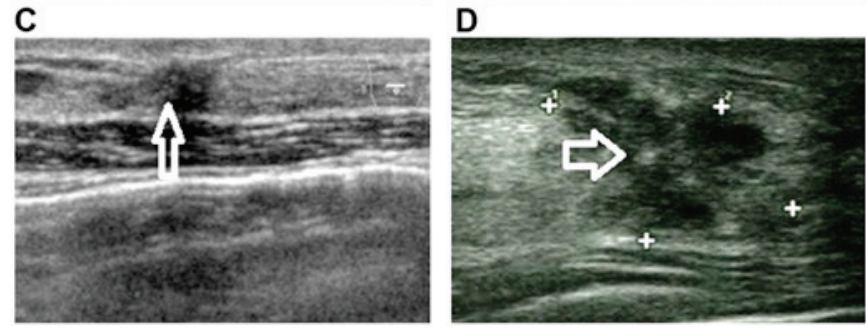

Figure 2. Malignant signs on imaging. (A) Hypoechoic mass with an irregular shape, indistinct and angled margin [Breast Imaging Reporting and Data System (BI-RADS) category 4C], which was confirmed as complex sclerosing adenosis (SA). (B) Heterogeneously echogenic area with rich blood supply (BI-RADS 4B), confirmed as SA with ductal epithelial hyperplasia. (C) Burr-like mass with diffusely punctate calcifications (arrow) (BI-RADS 4B), diagnosed as SA with calcium deposition. (D) Irregular mass with angled margin and clustered amorphous calcifications (arrow) (BI-RADS 4B), confirmed as SA with atypical ductal hyperplasia and calcium deposition.

rate, defined as the percentage of lesions with an indication for clinical intervention (BI-RADS categories 0 and 4A-5) among the pathologically benign lesions, was $45.1 \%$ (60/133). Among false-positive lesions, 12 were accompanied by atypical ductal hyperplasia, 4 displayed calcium deposition microscopically, whereas 1 lesion had both. The false-negative rate, defined as the percentage of lesions indicated as benign (BI-RADS categories 1-3) among the pathologically malignant lesions, was $55.6 \%(10 / 18)$.

In the 14 lesions with heterogeneously echogenic areas, apart from 5 lesions classified as BI-RADS US categories 1-3, 4 required further examination (category 0 ), whereas the remaining 5 lesions were classified as category $4 \mathrm{~A}-4 \mathrm{~B}$, among 
Table III. Comparison of findings in sclerosing adenosis of the breast between ultrasonography (US) and mammography (MG).

MG findings

\begin{tabular}{lcccccc}
\cline { 3 - 6 } US findings & $\begin{array}{c}\text { Microcalcifications } \\
(\mathrm{n}=43)\end{array}$ & $\begin{array}{c}\text { Masses } \\
(\mathrm{n}=32)\end{array}$ & $\begin{array}{c}\text { Asymmetric } \\
\text { focal density } \\
(\mathrm{n}=20)\end{array}$ & $\begin{array}{c}\text { Focal architectural } \\
\text { distortion } \\
(\mathrm{n}=31)\end{array}$ & $\begin{array}{c}\text { Negative } \\
(\mathrm{n}=10)\end{array}$ & $\begin{array}{c}\mathrm{NA}^{\mathrm{a}} \\
(\mathrm{n}=15)\end{array}$ \\
\hline Heterogeneously echogenic areas $(\mathrm{n}=14)$ & 4 & 0 & 2 & 6 & 1 & 1 \\
Masses (n=78) & 18 & 21 & 8 & 16 & 5 & 10 \\
Masses with calcifications ( $\mathrm{n}=21)$ & 6 & 7 & 3 & 2 & 2 & 1 \\
Focal acoustic shadowing (n=6) & 0 & 0 & 4 & 0 & 2 & 0 \\
Negative (n=15) & 15 & 4 & 3 & 7 & 0 & 3 \\
\hline
\end{tabular}

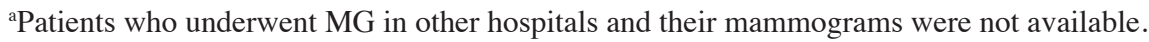

which only 2 lesions were finally confirmed as malignant. A total of $78.1 \%(25 / 32)$ of the US-negative lesions were simple SA histopathologically, without accompanying malignant or benign lesions.

Focal acoustic shadowing had been described in previous studies $(5,6)$; ours included 6 lesions $(4.0 \%)$, with 1 lesion classified as BI-RADS US category 0,1 as $4 \mathrm{~A}$, and 4 as $\geq 4 \mathrm{~B}$, but only 1 lesion exhibiting focal acoustic shadowing was finally histopathologically confirmed as SA with malignant lesions. Although considered as a sign of malignancy, focal acoustic shadowing was not found to be significantly correlated with histopathological malignancy $(\mathrm{P}=0.519)$.

In the 60 lesions that were overestimated by BI-RADS US category, one or more characteristics of malignancy were found in US imaging, such as irregular shape in 54 lesions (90.0\%), ill-defined margins in 46 lesions (76.7\%), calcifications in 12 lesions (20\%), focal acoustic shadowing in 6 lesions (4.0\%) and hypervascularity in 7 lesions (11.7\%) (Fig. 2).

$M G$ findings and comparison with US. A total of 136 patients underwent MG in our center and abnormalities were detected in 126 patients $(92.6 \%)$, whereas the examination was negative in 10 patients $(7.4 \%)$. There were mainly four types of imaging findings: Microcalcifications $(n=43 ; 31.6 \%)$, masses $(n=32$; $23.5 \%)$, asymmetric focal density $(n=20 ; 14.7 \%)$ and focal architectural distortion $(\mathrm{n}=31 ; 22.8 \%)$.

Of the 43 microcalcifications, 34 (79.1\%) were clustered, $8(18.6 \%)$ were scattered and $1(2.3 \%)$ was diffuse. The shapes of microcalcifications were punctate in 29 (67.4\%), round in $5(11.6 \%)$, pleomorphic in $7(16.3 \%)$, and amorphous in $2(4.7 \%)$ cases. On US imaging, $15(34.9 \%)$ of the MG microcalcifications were invisible, $6(14.0 \%)$ were masses with calcifications and the remaining were lesions without calcifications, including $4(9.3 \%)$ heterogeneously echogenic areas and 18 masses $(41.9 \%)$.

Unlike US imaging, on which masses and masses with calcificaitons constituted the majority $(65.6 \%)$, masses were only found in $32(23.5 \%)$ cases in MG. Of the 6 lesions with focal acoustic shadowing on US, 2 were negative on MG and 4 exhibited as asymmetric focal density (Table III).

Comparing BI-RADS MG category with histopathology, the AUC of MG distinguishing between benign and malig- nant lesions was significantly smaller compared with US (0.497 vs. 0.547 , respectively; $\mathrm{P}=0.036$ ).

\section{Discussion}

SA is a benign proliferative disease of the epithelium and myoepithelium that originates in glandular lobules and is accompanied by desmoplasia. Although it is a benign disorder, SA may prove challenging in both clinical and radiological aspects, as it may mimic malignancy grossly and microscopically. Furthermore, SA is occasionally complicated by malignant tumors, such as IDC and DCIS, and certain benign disorders, such as fibroadenosis, intraductal papilloma and atypical ductal hyperplasia, which makes differential diagnosis more difficult prior to surgery.

Although SA was not considered as a precancerous lesion, a higher risk of breast carcinoma has been reported in patients with SA $(10,11)$. In a cohort of 13,434 women followed up for a median of 15.7 years, Visscher et al (12) found that the presence of SA stratified risk in subsets of women defined by age, involution status and family history, and that SA is a common proliferative lesion of the breast that conveys an approximate doubling of breast cancer risk.

In the present study, malignant lesions were identifies in 20 of the 151 lesions (13.4\%), among which 13 lesions were DCIS (SA DCIS), 4 were DCISM and 3 were IDC. Among the patients with SA DCIS, 1 patient was confirmed as synchronous bilateral breast cancer. In previous studies, Moritani et al (13) reported 5 cases of synchronous or metachronous contralateral breast carcinoma developing among 23 patients with SA DCIS; Yoshida et al (14) reported a significantly higher rate of synchronous and metachronous bilateral breast cancer in SA DCIS compared with non-SA DCIS [9 $(38 \%)$ of 24 patients vs. $22(13 \%)$ of 174 patients; $\mathrm{P}<0.01]$. A retrospective study in our center previously demonstrated that the presence of SA was an independent risk factor for synchronous bilateral breast cancer $(\mathrm{P}<0.001)(15)$. Cancer genuinely arising in SA may often have biological characteristics of bilateral breast cancer (16), thus making it more important to distinguish it through radiological examinations.

US is generally accepted as an efficient method that is most commonly used in breast diseases, due to its advantages 
of non-invasiveness and convenience; in addition, its clearer imaging of dense breast tissue compared with MG makes it more popular in Eastern countries. However, previous studies mostly focused on the MG findings of SA, while those of US have rarely been discussed (3-5). The results of MG findings in our study were almost consistent with previous studies, which revealed mainly four types of imaging, with microcalcifications as the most common type. Although MG was more sensitive for discovering abnormalities compared with US in our study, the AUC of US distinguishing benign from malignant lesions was larger compared with that of MG $(0.547$ vs. 0.497, respectively; $\mathrm{P}=0.036$ ).

In the present study, 151 lesions in 145 patients were detected by US, and SA was histopathologically confirmed as the major component. Mass lesions without calcification constituted the major component among all cases (78 lesions; 51.7\%), more frequently compared with MG (32 lesions; 23.5\%), which was consistent with previous reports $(12-44 \%)(5,6)$. The majority of the visible lesions were irregular in shape (58.0\%) and had an ill-defined margin (52.1\%), which were probable indications of malignancy; this may be one of the reasons for the overestimation of BI-RADS US category in certain cases. Likewise, hypervascular lesions were all classified as category $\geq 4$, indicating a malignant tendency to a certain degree. Only few of the previous studies had described the US characteristics, so we herein compared our results with those of MG. However, the morphology of masses detected by MG, including shape and margin, did not lead to the same conclusion in each study (4-6), possibly as a result of the small samples, whereas, to the best of our knowledge, our sample was the largest thus far. However, the shape and margin of the lesions were not significantly different among simple SA, SA with malignant lesions and SA with benign lesions, which may be attributed to the variable pathological manifestations of SA $(1,13,17)$.

Microcalcifications were found to be one of the major characteristics of SA on MG (6), with $80 \%$ of the cases presenting as microcalcifications in clusters and $20 \%$ being diffusely scattered. Only 1 case of clustered calcifications without a mass was found through US in our study, in which SA was the minor component and DCIS was the major component. Of the 151 lesions with SA as the major component, masses with calcification were found in $21(13.9 \%)$ cases on US; the most frequent calcification pattern was clustered punctate and the remaining were diffusely punctate and clustered amorphous calcifications. However, the presence of calcifications was not significantly correlated with histopathology, which may be attributed to the hyposensitivity of US for identifying calcifications.

In addition to masses with or without calcification, there were quite a few cases [14 (9.3\%)] presenting as heterogeneously echogenic areas on US images; focal architectural distortion of breast tissue without occupying effect was the characteristic imaging finding. There were also 32 lesions (21.2\%) without visible abnormalities on US, $78.1 \%$ of which (25/32) were simple SA histopathologically, without accompanying malignant or benign lesions. Thus, simple SA more frequently presented as negative on US, and patients with this type of SA partly contributed to the false-negative rate.

The difficulty of diagnosing SA through US also manifested as the relatively high false-positive rate, which was
$45.1 \%$ in our study. As mentioned above, certain US characteristics of malignancy, such as calcifications, irregular shape, indistinct margins, focal acoustic shadowing and hypervascularity, contributed to the overestimation of diagnosis (Fig. 2). As reported in studies on fine-needle aspiration cytology of SA, the most frequent characteristics, although not specific, were low-to-moderate cellularity, bland epithelial cells that focally formed cohesive groups/tubules or occasionally discohesive clusters or individual cells, and fragments of dense fibrous stroma (18). Some tubules had an angulated configuration. These histopathological characteristics might contribute to the malignant signs of SA on imaging examination.

In conclusion, the accuracy of US in diagnosing SA was limited, although it was significantly higher compared with MG. Four types of US presentation were also observed in our series and compared with MG; to the best of our knowledge, the present study was the first to discuss these findings in detail to date. The high frequency of malignant signs on US may contribute to the misdiagnosis of SA. These findings may prove helpful for further investigation, which should focus on contrast-enhanced US and shear wave elastography and may improve the accuracy of diagnosis.

\section{Acknowledgements}

The present study was supported by the National Natural Science Foundation of China (grant no. 81371575).

\section{References}

1. Tavassoli FA: Pathology of the Breast. Appleton and Lange, Norwalk, CT, pp93-97, 1992.

2. Santen RJ and Mansel R: Benign breast disorders. N Engl J Med 353: 275-285, 2005.

3. Nielsen NS and Nielsen BB: Mammographic features of sclerosing adenosis presenting as a tumor. Clin Radiol 37: 371-373, 1986.

4. DiPiro PJ, Gulizia JA, Lester SC and Meyer JE: Mammographic and sonographic appearances of nodular adenosis. AJR Am J Roentgenol 175: 31-34, 2000.

5. Günhan-Bilgen I, Memiş A, Ustün EE, Ozdemir N and Erhan Y: Sclerosing adenosis: Mammographic and ultrasonographic findings with clinical and histopathological correlation. Eur J Radiol 44: 232-238, 2002.

6. Taşkin F, Köseoğlu K, Unsal A, Erkuş M, Ozbaş S and Karaman C: Sclerosing adenosis of the breast: Radiologic appearance and efficiency of core needle biopsy. Diagn Interv Radiol 17: 311-316, 2011.

7. Levy L, Suissa M, Chiche JF, Teman G and Martin B: BIRADS ultrasonography. Eur J Radiol 61: 202-211, 2007.

8. Mercado CL: BI-RADS update. Radiol Clin North Am 52: 481-487, 2014

9. Adler DD, Carson PL, Rubin JM and Quinn-Reid D: Doppler ultrasound color flow imaging in the study of breast cancer: Preliminary findings. Ultrasound Med Biol 16: 553-559, 1990.

10. Kabat GC, Jones JG, Olson N, Negassa A, Duggan C, Ginsberg M, Kandel RA, Glass AG and Rohan TE: A multi-center prospective cohort study of benign breast disease and risk of subsequent breast cancer. Cancer Causes Control 21: 821-828, 2010.

11. Hartmann LC, Sellers TA, Frost MH, Lingle WL, Degnim AC, Ghosh K, Vierkant RA, Maloney SD, Pankratz VS, Hillman DW, et al: Benign breast disease and the risk of breast cancer. N Engl J Med 353: 229-237, 2005.

12. Visscher DW, Nassar A, Degnim AC, Frost MH, Vierkant RA, Frank RD, Tarabishy Y, Radisky DC and Hartmann LC: Sclerosing adenosis and risk of breast cancer. Breast Cancer Res Treat 144: 205-212, 2014. 
13. Moritani S, Ichihara S, Hasegawa M, Endo T, Oiwa M, Shiraiwa M, Nishida C, Morita T, Sato Y, Hayashi T, et al: Topographical, morphological and immunohistochemical characteristics of carcinoma in situ of the breast involving sclerosing adenosis. Two distinct topographical patterns and histological types of carcinoma in situ. Histopathology 58: 835-846, 2011.

14. Yoshida A, Hayashi N, Akiyama F, Yamauchi H, Uruno T, Kikuchi M, Yagata H, Tsugawa K, Suzuki K, Nakamura S and Tsunoda H: Ductal carcinoma in situ that involves sclerosing adenosis: High frequency of bilateral breast cancer occurrence. Clin Breast Cancer 12: 398-403, 2012.

15. Chen JJ, Wang Y, Xue JY, Chen Y, Chen YL, Xiao Q, Yang WT, Shao ZM and Wu J: A clinicopathological study of early-stage synchronous bilateral breast cancer: A retrospective evaluation and prospective validation of potential risk factors. PLoS One 9 e95185, 2014.
16. Ogura K, Horii R, Oosako T, Iwase T and Akiyama F: A clinico-pathological study on cancer in sclerosing adenosis. Breast Cancer 21: 732-737. 2014.

17. Fechner RE: Carcinoma in situ involving sclerosing adenosis. Histopathology 28: 570, 1996.

18. Kundu UR, Guo M, Landon G, Wu Y, Sneige N and Gong Y: Fine-Needle aspiration cytology of sclerosing adenosis of the breast: A retrospective review of cytologic features in conjunction with corresponding histologic features and radiologic findings. Am J Clin Pathol 138:96-1028, 2012. 